\title{
雄性多音天蚕和柞蚕触角短毛感器中的 第三种嗅觉感受细胞*
}

\author{
吴才宏 \\ (北京大学生物学系)
}

\author{
H. J. Bestmann
}

（联邦德国埃尔兰根一纽伦堡大学有机化学研究所）

\section{关钺词毛形感器、感受细胞、信息素、多音天盉}

在鳞翅目昆虫中, 毛形感器已被公认为性信息素接收器. 多音天寔和柞亘的雄蛾触角至 少存在两种长短不同的毛形感器, 分别称为长毛感器和短毛感器. Kochansky 等鉴定了多音 天亘性信息素的两个组分为反-6, 顺-11-十六碳二烯醇醋酸酯 (E-6, Z-11-16:Ac) 及反-6, 顺-11-十六碳二烯酫（E-6, Z-11-16: $\left.A_{i}\right)^{[1]}$; Bestmann 等鉴定了上述两种化合物及反-4, 顺-9-十四碳二烯醇醋酸酯 (E-4, Z-9-14!Ac) 为柞蚠性信息素的三个组份 ${ }^{[2]}$. Kaissling 发 现多音天查和柞亘的长毛感器中存在两种不同类型的感受细胞, 分别对 E-6, Z-11-16:Ac 及 E-6, Z-11-16: Al 两种化合物具有特异的敏感性 ${ }^{[3]}$. Steinbrecht 在形态学研究中, 发现雄性 柞蚕触角的长毛感器中存在 $1-3$ 个感受细胞 ${ }^{[4]}$. Löfstedt 等发现黄地老虎雄蛾触角的毛形 感器中存在 4 种不同类型约感受细胞, 而且证明 4 种细胞的分布是不均匀的 ${ }^{[s]}$.

本文用电生理学方法发现, 在雄性多音天寔和柞蚕触角的短毛感器中, 存在对 E-4, Z-914:Ac 敏感的第三种感受沺胞, 并研究了三种细胞在短毛感器中的分布. 以期进一步探讨和 确证这些感受细胞和信息素系统各组分之间的关系,结合行为和模型研究, 译释不同化学组份 在化学通讯中的作用.

\section{一、材料与方法}

本实验以多音天素和泎亘为材料. 新鲜蚕茧在 $0^{\circ} \mathrm{C}$ 左右的冷库中保存. 每周从冷库中取 出少许毛茧, 雌、雄分离, 於:置在室温下, 2-3 周后即可羽化, 一般用羽化后 2-3 天内的雄蛾 触角作为实验材料.

本实验用单个感受细胞记录的方法, 从未交配的雄性柞亘和多音天蚕触角的短毛感器记 录到感受器电位和神经脉冲. 切下触角的一个侧枝, 参考电极插人侧枝的开放端, 用一特制的 镊子切去侧枝上几个短毛感器的尖端, 记录电极套在一根切去顶端的短毛上. 玻璃毛细管电 极充满盐液, 用 $\mathrm{Ag}-\mathrm{AgCl}$ 引导. 盐液的离子成分参照 Kaissling ${ }^{[6]}$ 的配方. 为防止感受器淋 巴液和电解质析出及电极和嗅觉毛的干燥, 电极尖端吸人少许 PVP 溶液 (用 $5 \mathrm{ml}$ 感受器淋巴

本文 1988 年 5 月 3 日收到. 1988 年 11 月 4 日收到修改稿.

*国家目然科学基金和联邦德国科协资助项目. 
液溶解 $1 \mathrm{~g}$ 聚乙烯吡咯烷酮), 在电极尖端周围涂以少许凡士林.

用正已烷稀释的气味化合物滴在 $15 \times 7 \mathrm{~mm}^{2}$ 的滤纸上，滤纸放在一端磨口的玻璃管内， 磨口端(入口)连接气流系统，出口对准触角的侧枝. 刺激期间由一电磁惐控制．两次刺激间， 隔为 $2 \mathrm{~min}$ 。通过样品管的气流量为 $100 \mathrm{ml} / \mathrm{min}$.

\section{二、结 果}

实验中, 每个触角远, 用三个侧枝, 每个侧枝选用一个具有自发脉冲发放的短毛感器作单细 胞记录, 共记录了多音天亘的 100 个短毛感器, 柞掻的 31 个短毛感器. 用 E-6,Z-11-16:Ac, E-6, Z-11-16: Al 及 E-4, Z-9-14:Ac 作为刺激化合物, 对上述三种化合物具有特异敏感性 的感受细胞分别被称为 $\mathrm{AC}_{1} 、 \mathrm{AI}$ 及 $\mathrm{AC}_{2}$ 型细胞. 刺激强度分别为 $2 \times 10^{-4} 、 2 \times 10^{-3}$ 及 $2 \times 10^{-2} \mu \mathrm{g}$.

\begin{tabular}{|c|c|c|}
\hline 细胞类型 & 刺激化合物 & 结 构 式 \\
\hline $\mathrm{AC}_{1}$ & $E-6, Z-11-16: A c$ & \\
\hline $\mathbf{A L}$ & $\mathrm{E}-6, \mathrm{Z}-11-16: \mathrm{Al}$ & \\
\hline $\mathrm{AC}_{4}$ & $\mathrm{E}-4, \mathrm{Z}-9-14: \mathrm{AC}$ & \\
\hline
\end{tabular}

图 1 多音天亘和柞蚕的性信息素组份及具有特异敏感性的感受细胞 E-6, Z-11-16:Ac 及 E-6, Z-11-16:Al 为两个种共有的性信息素组份; E-4,Z-9-14:Ac 为柞盖珄信息索的第三种组份
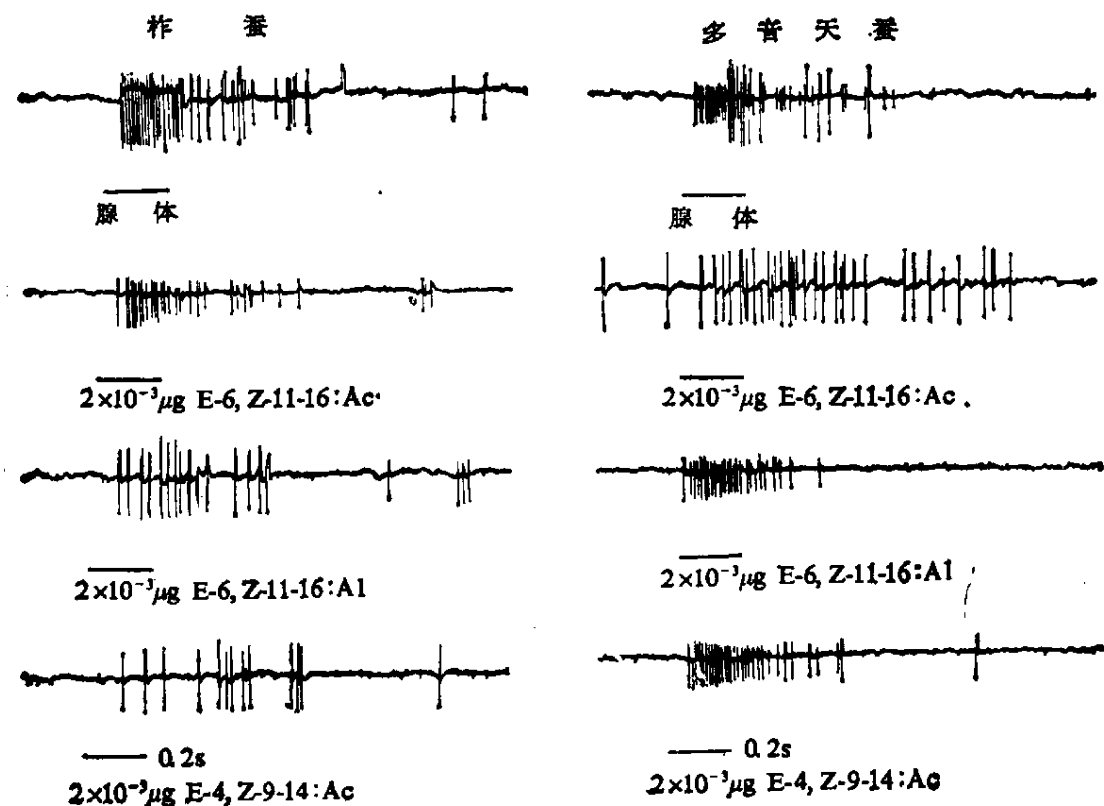

$2 \times 10^{-3} \mu \mathrm{g}$ E-4, Z-9-14:Ac

图 2 雄性柞亘和多音天亘触角短毛感器的单细胞记录

第一护为两个种的短毛感器对相应种的㫿娥腺体的反应; 第二至第四排分别为对 E-6, Z-11-16: AC、E-6, Z-11-16: A1 及 E-4, Z-9-14: Ac 的反应. 刺潡 期间为 $200 \mathrm{~ms}$ ，谪在誌纸上的刺激化合物量为 $2 \times 10^{-3} \mu \mathrm{g}$ 
实验中发现,多音天寔和柞䖯触角的短毛感器中, 除了存在对 E-6, Z-11-16:Ac 及 E-6, Z-11-16: $\mathrm{Al}$ 具有特异敏感性的 $\mathrm{AC}_{1}$ - 型及 $\mathrm{AL}$-型细胞外, 还存在对 $\mathrm{E}-4, \mathrm{Z}-9-14: \mathrm{Ac}$ 具有 特异敏感性的 $\mathrm{AC}_{2}$-型细胞, 即为第三种嗅觉感受细胞. 图 2 表明, 柞虫触角短毛感器中的 $\mathrm{AC}_{1}$ - 型细胞对，E-6, Z-11-16: Ac 发放小脉冲, $\mathrm{AL}$-型细胞对 E-6, Z-11-16:Al 发放大脉 冲, $\mathrm{AC}_{2}$-型细胞对 E-4, Z-9-14: $\mathrm{Ac}$ 也发放大脉冲; 在多音天䖽, $\mathrm{AC}_{1}$-型细胞对 $\mathrm{E}-6, \mathrm{Z}-$ 11-16:Ac 发放大脉冲, AL-型细胞对 E-6, Z-11-16: $\mathrm{Al}$ 发放小脉冲, $\mathrm{AC}_{2}$-型细胞对 $\mathrm{E}-4$, Z-9-14:Ac 也发放小脉冲。短毛感器对于同种的䧳蛾腺体的反应均为发放大、小不同的两类 脉冲。对于两个种, $\mathrm{AC}$ - 型细胞和 $\mathrm{AL}$-型细胞发放的脉冲振幅不同,是属于两种不同类型的 嗅觉感受细胞. $\mathrm{AL}-$ 型与 $\mathrm{AC}_{2}$-型细胞发放的脉冲振幅相同, 是否属于同一类型的细胞呢? 可 用适应性试验加以证明 ${ }^{[7]}$. 如图 3 所示, 先用强度为 $2 \times 10^{-2} \mu \mathrm{g}$ 的 E-4, Z-9-14:Ac 作为 条件刺激,紧接着用强度为 $2 \times 10^{-3} \mu \mathrm{g}$ 的 E-6, Z-11-16:Al 作为试验刺激, 先后记录到两 个电反应. 条件刺激强序比试验刺激强度高 10 倍, 而试验刺激的反应比条件刺激所引起的反 应更大, 说明 $\mathrm{AL}$ - 型细胞!并不能为条件刺激所适应, 从而证明 $\mathrm{AL}$-型和 $\mathrm{AC}_{2}-$ 型细胞是属于 不同类型的嗅觉感受细胞. 在两个种的绝大多数长毛感器中, 对于 $\mathrm{E}-6, \mathrm{Z}-11-16: \mathrm{Al}$ 具有特 异敏感性的 AL-型细胞㳔于 E-4, Z-9-14:Ac 却无反应, 从另一个侧面证明 AL-型细胞是

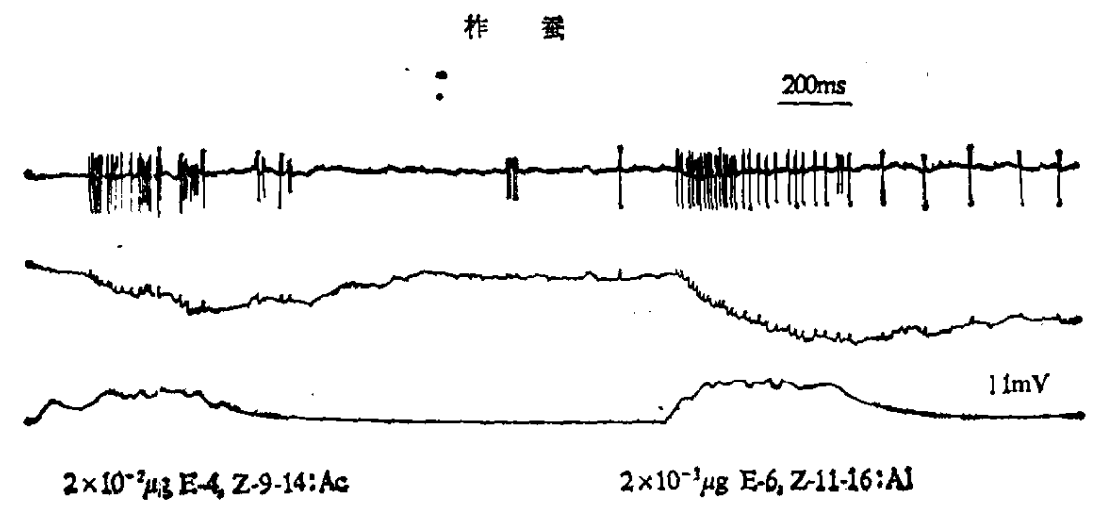

图 3 雄性柞蚕触角短毛感器中两种类型感受细胞的电反应

上排为交流偶合的神经脉冲反应; 中排为直流偶合的感受器电位, 脉冲反应叠加在感受器电位上;

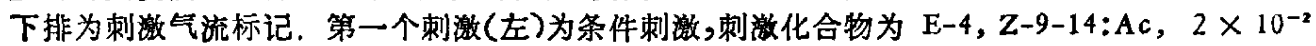

$\mu \mathrm{g}$; 第二个刺激、右)为试验刺激, $2 \times 10^{-3} \mu \mathrm{g}$ 的 E-6,Z-11-16:A1, 刺激期间为 $200 \mathrm{~ms}$

表 1 雄性多音天宴和柞䖯触角的短毛感器对三种化合物的反应

\begin{tabular}{|c|c|c|c|c|c|c|}
\hline \multirow{3}{*}{ 反 } & \multicolumn{3}{|c|}{ 多音 天 亘 } & \multicolumn{3}{|c|}{ 柞 貝 } \\
\hline & \multicolumn{3}{|c|}{ 化 合 物 } & \multicolumn{3}{|c|}{ 化 合 物 } \\
\hline & $E-6, Z-11-16: A c$ & E $-4, Z-9-14: A c$ & $E-6, Z-11-16: A L$ & $\begin{array}{l}\mathrm{E}-6, \mathrm{Z}-11- \\
16: A \mathrm{c}\end{array}$ & $\begin{array}{l}\text { F-4, Z-9- } \\
14: A C\end{array}$ & $\begin{array}{l}\text { E-6, Z-11- } \\
16: A L\end{array}$ \\
\hline$+t+$ & 16 & 29 & 5 & 5 & 4 & 23 \\
\hline++ & 80 & 62 & 26 & 23 & 6 & 7 \\
\hline+ & 1 & 2 & 13 & 3 & 7 & 0 \\
\hline - & 3 & 7 & 56 & 0 & 14 & 1 \\
\hline$\%$ & $97 \%$ & $93 \%$ & $44 \%$ & $100 \%$ & $55 \%$ & $97 \%$ \\
\hline
\end{tabular}

表中前三行数字为对三种化合物有反应的感受细胞数, 第四行为无反应的感受细胞数, 最后一行为有反应的感器占所 淴感器的百分数。 +++ 表示对 $2 \times 10^{-4} \mu \mathrm{g}$ 的化合物有反应, ++ 表示对 $2 \times 10^{-3} \mu \mathrm{g}$ 的化合物有反应, + 表示对 $2 \times 10^{-2} \mu \mathrm{g}$ 的化合物有反应, 一 表示对三种强度的化合物均无反应.

第 8 胡

科通报 
不同于 $\mathrm{AC}_{2}$-型细胞.

本实验所记录的多音天㔬触角的 100 个短毛感器中, 对 E-6, Z-11-16:Ac 有反应的 $\mathrm{AC}_{\mathrm{1}}$ - 型细胞有 97 个, 对 E-6, Z-11-16: Al 有反应的 AL-型细胞有 44 个, 对 E-4, Z-9-14:Ac 有反应的 $\mathrm{AC}_{2}$ - 型细胞有 93 个,有反应的细胞总数共为 234 个. 在所记录的柞亘触角的 31 个 短毛感器中, $\mathrm{AC}_{1}$ - 型细胞为 31 个, $\mathrm{AL}$-型细胞为 30 个, $\mathrm{AC}_{2}$-型细胞为 17 个, 有反应的细 胞总数为 78 个. 气味刺激以后产生 10 个以上的神经脉冲即为有反应, 表 1 说明, 对于多音天 䖽, $\mathrm{AC}_{2}$-型细胞占有反应细胞总数的 $39.9 \%, \mathrm{AC}_{1}$ - 型细胞占 $47.5 \%, \mathrm{AL}$ - 型细胞占 $18.8 \%$; 对 于柞寔, $\mathrm{AC}_{2}$ - 型细胞占 $21.8 \%, \mathrm{AL}$ - 型细胞占 $38.8 \%, \mathrm{AC}_{1}$-型细胞占 $39.7 \%$ 。 上述数据表明, 在雄性多音天亘和柞毛触角的短毛感器中, 均存在相当数量的对 E-4, Z-9-14:Ac 敏感的第 三种感受细胞.

\section{三、讨 论}

昆虫性信息素系统的各组分含量是以一定的比例存在于堆蛾的腺体中. 多音天毛雌蛾腺 体中, E-6, Z-11-16:Ac:E-6, Z-11-16:Al = 90:10 ${ }^{[1]}$, 尚未发现有 E-4, Z-9-14:Ac. 柞 亘䧼蛾腺体中含有 E-6, Z-11-16:Ac、E-6, Z-11-16:AI 及 E-4, Z-9-14:Ac 三个组分, 其 比例为 $20: 100: 40^{[2]}$. 在两个种的雄蛾触角的短毛感器中, 均存在对 E-4, Z-9-14:Ac 敏感 的第三种感受细胞. 上述现象可能是进化过程中形成的种间差异, 对于种间隔离可能是有意义 的. 雌蛾腺体中性信息素系统各组分间的比例与雄蛾触角的接收系统中不同类型感受细胞的 数量分布及组合状态, 对于昆虫种内和种间的化学通讯起重要的作用. 本研究对于译释这种 化学语言提供了某些启示,但尚需进一步结合形态学及行为反应进行综合研究.

在雄性多音天亘和柞蚕触角的长毛感器中, 大多数情况, 只是在 E-4, Z-9-14:Ac $\left(2 \times 10^{2}\right.$ $\mu \mathrm{g}$ ）高刺激强度时才能引起多音天亘发放大脉冲, 柞峑发放小脉冲, 可以认为是 $\mathrm{AC}_{1}$ - 型细胞 的反应 ${ }^{[8]}$. 只是在极少数的情况下, $2 \times 10^{-3} \mu \mathrm{g}$ 的 E-4, Z-9-14:Ac 能使少数长毛感器产 生以下反应: 多音天蚕发放小脉冲, 柞亘发放大脉冲, 可以认为这是 $\mathrm{AC}_{2}$-型细胞的反应, 且 $\mathrm{AC}_{2}$ - 型细胞在长毛感器中的分布是非常稀少的. 因此, 要比较客观地反映 $\mathrm{AC}_{2}$-型细胞在长 毛感器中的分布状况, 只记录几十个长毛感器是没有意义的, 必须记录成干个长毛感器才有意 $义^{[3]}$.

\section{参考文献}

[ 1 ] Kochansky, J. et al., J. Insect Physiol., 21(1975), 1977-1983.

[2] Bestmann, H. J. et al., Z. Naturforsch, 42c(1987), 631-636.

[3] Kaissling, K. E., Insect Olfaction, Simon Fraser Unversity, Burnaby, B. C., Canada, 1987.

[4] Steinbrecht, R. A. et al., Cell Tissue Res., 235(1984), 25-34.

[ 5 ] Löfstedt, C. et al., J. Chem. Ecol., 10(1982); 1305-1321.

[6] Kaissling, K. E., Receptors for Neurotransmitters, Hormones and Phercomones in Insect, Elsevier/North-Holland Biomedical Press, 1980.

[7] Kaissling, K. E., Int. Sym. Olfaction and Tastc IX, N. Y. Acad. Sci., 1987.

[ 8 ] Bestmann, H. J., Wu, C. H. et al., Z. Naturforsch, 42c(1987), 435-441. 